\title{
Searching for Low-Mass Companions with the HST WF/PC
}

\author{
WILLIAM A. BAUM \\ Astronomy Department, University of Washington, \\ Seattle, WA 98195, USA
}

\begin{abstract}
Owing to the spherical aberration of the Hubble Space Telescope, prospects are now poor for the astrometric detection of low-mass companions of nearby stars with the Wide-Field / Planetary Camera. The installation of a new camera (WF/PC-II) incorporating an optical correction for the telescope will only partially recover the low-mass companion search capability.
\end{abstract}

\section{INTRODUCTION}

Early observations with the Wide-Field / Planetary Camera (WF/PC) aboard the Hubble Space Telescope (HST) were originally planned by the WF/PC team to include images of very nearby stars believed suitable for close inspection and for monitoring astrometrically over the lifetime of the HST (Baum 1980). Due to the severe spherical aberration of the primary mirror of the HST, that plan has largely been put on hold, pending the launch of a replacement camera (WF/PC-II) which will incorporate an optical correction for the telescope aberration. Moreover, during the 15 years since the WF/PC team's scientific agenda first took shape, improvements in groundbased astrometry have reduced the zeal for using WF/PC to carry out an astrometric search for low-mass companions of nearby stars. The purpose of this paper is to offer comments on future prospects.

\section{THE WF/PC}

The WF/PC has been described in various publications and documents (e.g., see Westphal et al. 1982; Griffiths 1990). After passing through a selected color filter, incoming light arrives at a four-faced reflecting pyramid which splits the HST cassegrain image into four quadrants. Each of the quadrants is relayed optically to a cooled $800 \times 800$-pixel CCD with a choice of two magnification modes. The Wide-Field Camera (WFC) mode provides an image scale of 0.1016 arcsec per pixel, and the Planetary Camera (PC) mode provides a scale of 0.0444 arcsec per pixel. The splitting of the image is relatively sharp, because the pyramid lies in the vicinity of the cassegrain focus of the telescope. There are no noticeable gaps in field coverage at chip boundaries. Even so, astrometric integrity is not well preserved across those boundaries, and astrometry is best confined to the fields of single chips. Owing to unused rows and columns along chip boundaries, the available area of each chip is roughly $770 \times 770$ pixels, which corresponds to $78 \times 78$ arcsec for each of the four WFC chips and $34 \times 34$ arcsec for each of the four PC chips.

The present imaging performance of the HST has been discussed in detail by Burrows et al. (1991). In the presence of spherical aberration, the point- 
spread function (PSF) depends on the location selected for the focal plane. The PSF at a location near the convergence of rays from the inner zones of the HST mirror is very different from the PSF near the convergence of rays from the outer zones. The "focal plane" (or more exactly, the focal surface) is, of course, defined by the entrance apertures and detectors of the various cassegrain instruments, including the WF/PC. After some experimenting, the telescope has been focused near the inner-zone convergence so as to produce a PSF having a sharp central spike containing $10 \%$ to $15 \%$ of the light, while the other $85 \%$ is distributed in wings that range out to more than $2^{\prime \prime}$. Those wings have tendrils and lumps (some of which look like faint stars) that are due to diffraction and that are wavelength dependent. Moreover, the PSF varies in a complicated way with its $(x, y)$ location on the chip. Astrometric measurements therefore depend on the fitting algorithm used. As if these problems were not enough, the telescope focus (and therefore the PSF) creeps with time, and it even cycles slightly during each 96-minute orbit.

The astrometric performance of the WF/PC has been discussed by Monet (1992) in the WF/PC team's Science Verification Report available from the Space Telescope Science Institute. For the WFC, the transformation between sky coordinates and pixel coordinates departs from a linear fit by 50 milliarcsec ( 0.5 pixel) or more, but residuals can be reduced to about 10 milliarcsec $(0.1$ pixel) by use of a cubic transformation. Although residuals are doubtless due in part to the slightly crinkly nature of the CCD surfaces, that component of the residuals will be difficult to map and will require far more observational data than are now available. In addition, quasi-random errors arise from the WFC undersampling of the core of the PSF, and simulations show that errors due to WFC undersampling would have been almost equally bad if the spherical aberration had not been present. This implies that WFC residuals will probably not be much improved by the installation of WF/PC-II.

Undersampling is less severe in the PC mode, but PC transformations and residuals have not yet been well determined.

In order to provide for favorable imaging of the fields surrounding nearby stars, an attenuating spot (generally known as the Baum Spot) was incorporated into the design of the WF/PC. The intention was that a bright star could be placed inside the spot so as to prevent it from saturating the CCD when the exposure is long enough to bring fainter stars in the surrounding background up to a usable signal level. Other intended uses of the spot originally included deep imaging of circumstellar disks and of fields surrounding the nuclei of galaxies. The Baum Spot produces a hole in all WF/PC images, but it is small enough (1".4 in diameter) to be relatively unobtrusive unless a bright object overlaps it. The spot is located on one of the faces of the WF/PC pyramid, and it consists of an antireflection coating on the quartz surface, seen through a well defined round hole in the aluminum overcoating.

Unfortunately, in the presence of the spherical aberration of the HST primary mirror, the PSF of a star centered inside the Baum Spot is so broad, bright, and lumpy that light splashing outside the spot totally precludes inspection of the immediate neighborhood of the star, and it severely interferes with the surrounding field of astrometric reference stars. The installation of $W F / P C$ II will not fix this problem, because optical correction of the spherical aberration is necessarily introduced at a pupil plane, which occurs later in the light path 
inside WF/PC-II.

As a result, the list of nearby stars that can be favorably dealt with by HST imaging is very much reduced and will remain so. Of the ten astrometric candidates in the WF/PC team's original list in Table 1, only three stars did not call for use of the Baum Spot. Those three (vB10, LFT544, and vB8) may remain viable candidates if a WF/PC search for low-mass companions is judged to be worth pursuing (either by direct inspection or by astrometric monitoring), and we note that vB10 has already been used for astrometric tests in connection with the HST Science Verification Program (Monet 1992).

TABLE 1. Original Target List for a WF/PC Search

\begin{tabular}{|lccccc|}
\hline \multicolumn{1}{|c}{ Star } & V mag & Spectrum & Parallax & Camera & Spot? \\
\hline Barnard & 9.5 & sdM5v & 0.552 & WFC & Yes \\
Ross 248 & 12.3 & dM6e & 0.318 & WFC & Yes \\
vB 10 & 17.4 & dM5 & 0.173 & PC & No \\
L726-8A,B & 12.5 & dM5.5e & 0.368 & WFC & Yes \\
Ross 154 & 10.6 & dM4.5e & 0.345 & PC & Yes \\
LFT 544 & 17.6 & M & 0.142 & WFC & No \\
Kruger 60B & 11.3 & dM4e & 0.253 & WFC & Yes \\
GRB 34B & 11.0 & M6Ve & 0.282 & WFC & Yes \\
L725-32 & 11.6 & M5e & 0.264 & WFC & Yes \\
vB 8 & 16.6 & $M$ & 0.161 & WFC & No \\
\hline
\end{tabular}

Several factors were taken into account in constructing the original list of astrometric candidates (Seidelmann et al. 1988). One can calculate the amplitude of the positional wobble that a hypothetical Jupiter would produce, but for an observing program of limited duration (say 10 years, as may be the case for the HST), the second derivative of the wobble may be more important than the amplitude of the wobble. Although we took these criteria into account, our final choices had to be based to a large degree on the availability of measurable backgound stars surrounding the program stars. Groundbased CCD images were therefore obtained at the USNO in Flagstaff, and final selections were made accordingly.

We have also become more aware of the degree to which astrometric detection indices depend on assumptions as to how planetary systems should scale with stellar mass. There is no physical basis for believing that the real world is represented by supposing orbital periods to be statistically similar from one planetary system to another, an assumption which underlies Gatewood's (1976) detection index. Nor is there any better basis for supposing orbital radii to be statistically similar from one planetary system to another, an assumption underlying our $\alpha$ index (Baum 1980). We have explored other assumptions (Baum 1989) that may have more physical justification, but the entire issue deserves further study. The point to emphasize here is that different models of planetarysystem scaling lead to different lists of preferred candidate stars. 


\section{ACKNOWLEDGMENTS}

Various members and associates of the HST WF/PC Investigation Definition Team participated in the planning of these observations. The team is currently supported by NASA Grant NAG 5-1611. The groundbased imaging survey of candidate targets was directed by P. K. Seidelmann and D. Pascu of the USNO.

\section{REFERENCES}

Baum, W.A. 1980, Celest. Mech., 22, 183

Baum, W.A. 1989, BAAS, 21, 973

Burrows, C.J., Holtzman, J.A., Faber, S.M., Bely, P.Y., Hasan, H., Lynds, C.R., \& Schroeder, D. 1991, ApJ, 369, L21

Gatewood, G. 1976, Icarus, 27, 1

Griffiths, R. 1990, in Wide Field Planetary Camera Instrument Handbook, (Baltimore, Space Telescope Science Institute)

Monet, D. 1992, in Final Orbital/Science Verification Report, ed. S. M. Faber (Baltimore, Space Telescope Science Institute), Chapter 7

Seidelmann, P.K., Pascu, D., \& Baum, W.A. 1988, in STScI Workshop on the Formation and Evolution of Planetary Systems, (Baltimore, Space Telescope Science Institute)

Westphal, J.A. and the WF/PC Investigation Definition Team 1982, The Wide-Field/ Planetary Camera, NASA CP-2244, The Space Telescope Observatory, ed. D.N.B. Hall (Washington, NASA Scientific and Technical Information Branch) p. 28

\section{DISCUSSION}

HARRINGTON: What is the current situation with respect to jitter for the camera?

BAUM: Jitter, if small in amplitude, is absorbed similarly into all PSF's within the field. Its effect in WF/PC astrometry may therefore be smaller than errors due to the crinkly nature of the CCD surfaces and errors due to WFC undersampling.

TAFF: The residuals in the positions of stars from the WF/PC resemble those seen in Schmidt plates. Hence, the methods recently invented to astrometrically reduce Schmidt plates might be profitably employed rather than the polynomial models heretofore employed.

BAUM: Problems associated with WFC undersampling and with time variation of the PSF's may make it difficult to characterize residuals well. Better data are needed than those now in hand.

ABT: Are the focus variations during the 96-min orbit predictable?

BAUM: In principle, yes. But it would not be a simple matter to apply that information to the assortment of PSF's that differ from one another with coordinate location, with spectral passband, and secularly with time. 